\title{
Détection de contaminants dans les huiles végétales : bilan à fin 2009
}

\author{
Florence LACOSTE \\ Florent JOFFRE \\ Jean-Louis COUSTILLE \\ Odile MORIN \\ Bénédicte SOULET \\ Evelyne BRENNE \\ Hugues GRIFFON \\ ITERG, \\ Institut des corps gras, \\ rue Monge, \\ 33600 Pessac, \\ France (www.iterg.com) \\ $<$ f.lacoste@iterg.com $>$
}

\begin{abstract}
The research of contaminants is part of multiple controls conducted by fat and oil industry to verify the conformity of products placed on the market in relation to regulations as for instance the commission regulation EC $n^{\circ} 1881 / 2006$ setting maximum levels for some contaminants in food as lead, some mycotoxins, dioxins, polychlorobiphenyls, benzo[a]pyrene. In the absence of regulation, the detection of contaminants must be addressed in partnership with authorities according to the toxicity of molecules. The controls are not confined to environmental contaminants. They also include compounds that can be formed during the production process of vegetable oils such as esters of 3-monochloropropanediol. This article focuses on recent research analytical development more particularly on those related to polycyclic aromatic hydrocarbons, mineral oils, phthalates and esters of 3-monochloropropanediol.
\end{abstract}

Key words: vegetable oils, polycyclic aromatic hydrocarbons, mineral oil, phthalates, 3-MCPD esters
La recherche de contaminants fait partie des multiples contrôles que doit réaliser l'industrie des corps gras pour vérifier la conformité des produits mis sur le marché par rapport aux réglementations en vigueur, comme par exemple le règlement européen $\mathrm{CE}$ $\mathrm{n}^{0} 1881 / 2006$ (Règlement CE, 2006). Celui-ci fixe des valeurs limites dans les huiles végétales pour: le plomb, certaines mycotoxines, les dioxines et les polychlorobiphényles, le benzo[a]pyrène (BaP). En l'absence de réglementation, la détection de contaminants doit être traitée en partenariat avec les instances publiques qui se positionnent en fonction de la toxicité des molécules.

Les contrôles ne se résument pas aux contaminants $d$ 'origine environnementale, mais englobent également les composés susceptibles de se former au cours du procédé d'obtention des huiles végétales, comme par exemple les esters de 3-monochloropropanediol.

\section{Hydrocarbures aromatiques polycycliques (HAP)}

\section{Contexte}

La présence des HAP dans les huiles végétales peut provenir de différentes sources, mais généralement les huiles contaminées sont issues de matières premières nécessitant d'être séchées telles que les pépins de raisin, le coprah ou les grignons d'olive.
À ce jour, huit composés de la famille des HAP sont classés cancérogènes de catégorie 2 par l'Union européenne: le BaP, le benzo [k]fluoranthène, le benzo[j]fluoranthène, le benzo[a]anthracène, le benzo[b]fluoranthène, le benzo[e]pyrène, le chrysène et le dibenzo $[a, h]$ anthracène (Directive CE, 2004). En se basant sur la conclusion du Comité scientifique européen sur les aliments (European Commission, 2002), qui considérait le BaP comme un marqueur de la présence des HAP les plus toxiques dans les aliments, la Commission européenne a réglementé en 2005 la teneur en BaP à une valeur maximale de $2 \mu \mathrm{g} / \mathrm{kg}$.

Pour l'huile de pépins de raisin, en 1994, la filière a connu une crise. La presse spécialisée allemande indiquait la présence d'HAP dans les huiles de pépins de raisin en provenance de France et d'Italie. En 2003, certains producteurs de ce type d'huiles brutes ont signalé des contaminations allant jusqu'à $1 \mathrm{mg} / \mathrm{kg}$ en $\mathrm{BaP}$, ces taux étant descendus, en 2004, à $30 \mu \mathrm{g} / \mathrm{kg}$ environ.

Pour les huiles de grignons d'olive, en 2001, I'Afssa recommandait de ne pas autoriser la commercialisation de certains lots de ces huiles venant $d^{\prime}$ Espagne. De plus, une étude menée en 2003 en Espagne faisait ressortir des contaminations allant jusqu'à $360 \mu \mathrm{g} / \mathrm{kg}$. Ces contaminations sont liées à un changement des procédés de fabrication, induisant la nécessité d'un séchage plus poussé.

Pour les autres huiles, il n'y a pas de contamination aussi importante. On trouve parfois des huiles de tournesol brutes à la limite du taux de BaP fixé par la réglementation européenne, soit $2 \mu \mathrm{g} / \mathrm{kg}$, mais bien inférieur aux cas cités précédemment. Des lots de tournesol d'origine ukrainienne sont fréquemment cités comme contaminés et sont souvent traités systématiquement avec des mélanges de terre décolorante et de charbon actif.

Une enquête publiée par la DG SANCO (2004) montre que les huiles pouvant dépasser la limite réglementaire de $2 \mu \mathrm{g} / \mathrm{kg}$ sont principalement les huiles de grignons d'olive, avec plus de $70 \%$ des échantillons "non conformes", les huiles de tournesol avec environ $30 \%$ d'échantillons «non conformes» et les huiles de pépins de raisin avec environ $16 \%$ d'échantillons « non conformes ».

La Commission européenne devrait courant 2010 modifier sa réglementation sur les HAP en intégrant d'autres molécules à surveiller, en plus du BaP. Elle se base notamment sur I'avis de l'Agence européenne de sécurité sanitaire des aliments (EFSA) du 9 juin 2008 (EFSA, 2008a), qui conclut que le BaP n'est pas un indicateur suffisant pour l'évaluation de la contamination en HAP des produits alimentaires, et qu'il convient d'étendre l'analyse à d'autres HAP. En effet, l'évaluation par l'EFSA de près de 10000 résultats de taux d'HAP dans différentes matières premières alimentaires, a démontré que le $\mathrm{BaP}$ pouvait être détecté dans environ $50 \%$ des échantillons. Toutefois, dans environ $30 \%$ de l'ensemble des échantillons, d'autres HAP 
cancérogènes et génotoxiques ont été détectés, malgré des tests négatifs pour le BaP.

L'EFSA a donc proposé à la Commission européenne, en tenant compte des données disponibles de cancérogénicité par voie orale, de retenir un groupe de huit ou un groupe de quatre HAP qui sont les suivants :

- «HAP8 »: BaP, chrysène, benz[a]anthracène, benzo[b]fluoranthène, benzo[k]fluoranthène, benzo[ghi]pérylène, dibenz $[a, h]$ anthracène et indéno[1,2,3-cd]pyrène ;

- « HAP4 »: BaP, chrysène, benz[a]anthracène et benzo[b]fluoranthène.

Les teneurs cibles pour ces HAP4 ou HAP8 ne sont pas encore connues.

\section{Méthode d'analyse}

Différents protocoles publiés récemment portent sur la détermination des HAP dans les corps gras. La préparation de l'échantillon fait intervenir une extraction liquide-liquide suivie d'une purification sur une colonne de silice (Moret et Conte, 2002), une colonne de silice puis une colonne de silice greffée aminopropyle (Moreda et al., 2004), une colonne de chromatographie d'exclusion stérique (Fiume et al., 2002), ou une colonne de chromatographie basée sur un complexe donneur-accepteur (Van Stijn et al., 1996). L'analyse de l'échantillon purifié est le plus souvent réalisée par HPLC-fluorescence.

II existe actuellement trois méthodes normalisées dans le domaine des corps gras qui portent soit sur le BaP, soit sur une dizaine de HAP :

- ISO 15032 : détermination de la teneur en $\mathrm{BaP}$ par HPLC-fluorescence après purification sur colonne d'alumine (ISO, 2007);

- ISO 15753 : détermination des HAP basée sur une extraction avec un mélange acétonitrile-acétone, suivie de purifications sur cartouches de silice greffée C18 puis Florisil $^{\circledR}$, et détermination par HPLCfluorescence (méthode développée par l'ITERG (ISO, 2006));

- ISO 22959: détermination des HAP par chromatographie de complexe donneuraccepteur d'électron (DACC), méthode basée sur une double chromatographie CLHP en ligne, la première sur colonne de type "donneur-accepteur » permettant d'isoler les HAP, la seconde sur silice greffée C18 et détection par fluorescence assurant l'analyse des HAP (ISO, 2009).

Cette dernière méthode est très utilisée au niveau de l'industrie des corps gras. Elle nécessite un équipement coûteux et une période de réglage de l'équipement, mais elle permet de limiter le nombre de manipulations et fournit des résultats en moins de deux heures.
L'ensemble des méthodes normalisées utilisent l'étalonnage externe et permettent d'atteindre le niveau de sensibilité prescrit par la réglementation $(2 \mu \mathrm{g} / \mathrm{kg})$, tant que l'échantillon ne présente pas de pics interférents, ce qui peut être le cas pour des matrices complexes comme les huiles de grignons d'olive.

\section{Traces d'huiles minérales}

\section{Contexte}

Les quelques publications (Neukom et al., 2002 ; Reich et al., 1997; Wagner et al., 2001; Moret et al., 2003) traitant de la présence $d$ 'hydrocarbures reportent des teneurs de l'ordre de quelques dizaines de milligrammes par kilogramme à environ une centaine de milligrammes par kilogramme dans les huiles végétales.

II faut cependant différencier les hydrocarbures naturellement présents dans les huiles végétales, qui sont des $n$-alcanes linéaires de longueur de chaîne inférieure à C40, des huiles minérales provenant d'une contamination, qui sont généralement des hydrocarbures ramifiés et cycliques. La réponse chromatographique obtenue pour ces deux types de composés est très différente. Pour les hydrocarbures naturels, des pics bien distincts sont obtenus. Pour les huiles minérales, de longueur de chaîne entre C20 et C50, la réponse chromatographique correspond à une courbe de Gauss formée par des pics non résolus (chromatogramme en forme de «bosse »).

Outre les risques de contamination par l'environnement (Neukom et al., 2002), les transports maritimes ou les fuites de fluides hydrauliques, il est également évoqué (Reich et al., 1997) I'utilisation d'huile minérale pour diminuer l'accumulation de poussière (dedusting) sur les graines oléagineuses lors de leur stockage. II semblerait que ce procédé soit admis aux États-Unis avec un niveau autorisé de 200 mg/kg (21 CFR 172.878).

Reich et al. (1997) ont compilé des données sur la présence d'hydrocarbures naturels dans environ 180 variétés végétales et aliments d'origine animale dont les teneurs pour graines oléagineuses et huiles végétales sont comprises entre 13 et $120 \mathrm{mg} / \mathrm{kg}$, la concentration la plus élevée étant enregistrée pour l'huile de tournesol. Les hydrocarbures naturellement présents dans les huiles végétales sont des n-alcanes linéaires de longueur de chaîne impaire. Pour l'huile de tournesol, les composés majoritaires possèdent 27,29 et 31 atomes de carbone. Pour I'huile d'olive, les composés majoritaires possèdent $23,25,27$ et 29 atomes de carbone. McGill et al. (1993) reportent des teneurs de l'ordre de 105 à $166 \mathrm{mg} / \mathrm{kg}$ pour des huiles de tournesol prélevées en GrandeBretagne, teneurs comparables à celles fournies par Reich et al.

Wagner et al. (2001) ont réalisé une étude sur la contamination des huiles végétales par les huiles minérales sur plus de 200 échantillons $\mathrm{d}^{\prime}$ huiles vierges et raffinées. Les concentrations varient entre 30 et $150 \mathrm{mg} / \mathrm{kg}$. Pour les huiles de tournesol (35 échantillons) et de colza (29 échantillons), les teneurs sont comprises entre 5 et $80 \mathrm{mg} / \mathrm{kg}$. Des essais de raffinage ont montré que la désodorisation éliminait les hydrocarbures de longueur de chaîne C25-C30, ce qui correspond généralement au tiers de la contamination, selon la nature de la contamination et les conditions de la désodorisation.

En octobre 1999, une contamination par du gazole (comprise entre 0,05 et $1,7 \%$ ) avait été constatée dans certaines cargaisons $d$ 'huile de palme brute d'origine indonésienne importées aux Pays-Bas. Ces cargaisons avaient été bloquées. Suite à des travaux d'analyse, il avait été démontré que l'huile de palme, après raffinage, ne contenait plus de gazole. L'ITERG, en liaison avec la Fédération nationale des industries de corps gras (FNCG) et FEDIOL avait également suivi ce dossier et développé une méthode pour quantifier la contamination. À la suite d'une contamination d'huiles brutes de tournesol en provenance d'Ukraine par des huiles minérales, des teneurs atteignant $7000 \mathrm{mg} / \mathrm{kg}$ dans les huiles brutes et $1100 \mathrm{mg} / \mathrm{kg}$ dans les huiles raffinées ont pu être mises en évidence, en 2008, en France. Dans d'autres pays de I'Union européenne (Grèce, Italie, Portugal, Espagne, Pays-Bas) les sources d'approvisionnement étant différentes, les taux de contamination pouvaient être différents de ceux constatés en France.

En relation avec la Fédération nationale des corps gras et les industriels concernés, I'ITERG a contribué à la vérification des lots incriminés ainsi qu'à la caractérisation de l'huile minérale en confiant pour analyse à I'Institut français du pétrole un échantillon obtenu après saponification de l'huile brute contaminée, puis isolement sur silice de la fraction des hydrocarbures. La distillation simulée de cet extrait a permis de vérifier les longueurs de chaîne de cette contamination, comprises entre C20 et C47. Les résultats ont montré que moins de $5 \%$ des composés de l'huile minérale avaient un point d'ébullition inférieur à $350^{\circ} \mathrm{C}$, ce qui a permis à l'Autorité européenne de sécurité des aliments (EFSA) de classer cette fraction comme " un type d'huile minérale de haute viscosité (mélange d'hydrocarbures paraffiniques et naphténiques liquides hautement raffinés) ».

Compte tenu de cette situation de crise, les industriels ont mis en place depuis mi-2008, 
une vérification systématique de tous les lots d'huile de tournesol importés. La Commission européenne $a$, depuis, publié un règlement relatif au contrôle des huiles de tournesol en provenance $d^{\prime} U$ kraine qui stipule que tout lot d'huile de tournesol présenté à l'importation doit être assorti d'un certificat attestant que le produit ne contient pas plus de $50 \mathrm{mg} / \mathrm{kg}$ de paraffine minérale (Règlement CE, 2009).

\section{Méthodes d'analyse}

L'ITERG a développé en 1999 une méthode $d^{\prime}$ analyse des huiles minérales dans les huiles végétales sur la base d'une publication de Tan et Kuntom (1993). Après dilution d'une prise d'essai de $2 \mathrm{~g}$ d'huile végétale dans $2 \mathrm{~mL}$ de $\mathrm{n}$-hexane contenant environ 0,1 mg d'octadécane (C18), la solution est éluée sur une colonne de $30 \mathrm{~g}$ de gel de silice de haute pureté par $150 \mathrm{~mL}$ de $\mathrm{n}$-hexane afin d'isoler la fraction des hydrocarbures. Après l'évaporation du solvant, l'extrait est repris dans $1 \mathrm{~mL}$ d'isooctane puis la détermination est effectuée par chromatographie en phase gazeuse (CPG) avec injecteur on-column, sur colonne capillaire de $15 \mathrm{~m}$ de phase stationnaire apolaire supportant des températures élevées (DB5 HT). La température du détecteur à ionisation de flamme est fixée à $380^{\circ} \mathrm{C}$. La programmation de la température du four, de $100{ }^{\circ} \mathrm{C}$ jusqu'à $370{ }^{\circ} \mathrm{C}$ à $20^{\circ} \mathrm{C} / \mathrm{min}$, permet d'éluer rapidement les huiles minérales et facilite ainsi l'intégration de l'enveloppe chromatographique. Les hydrocarbures (la plupart du temps n-alcanes) présents naturellement dans l'huile végétale ne sont pas pris en considération dans le calcul de la teneur de l'huile minérale (figure 1). La quantification est basée sur l'inté- gration de l'enveloppe chromatographique due à la présence d'huile minérale. La limite de la quantification est fixée à $50 \mathrm{mg}$ d'huile minérale par kilogramme d'huile végétale.

En fin d'année 2008, la Commission européenne a commissionné I'Institute for Reference Materials and Measurements (IRMM) du Joint Research Centre afin d'organiser un essai d'intercomparaison sur la détermination des huiles minérales dans I'huile de tournesol. L'objectif était d'identifier les points critiques de cette détermination mais aussi d'évaluer la performance des laboratoires officiels et privés qui ont réalisé des contrôles durant l'incident. Les échantillons confiés pour analyse étaient des huiles de tournesol (brute ou raffinée), contaminées en huile minérale. Les résultats de cet essai ont été communiqués par l'IRMM à la fin du mois d'avril 2009 (Karasek et al., 2009).

Au total, 55 laboratoires ont participé à cet essai. Les méthodes utilisées par les participants à cet essai sont diverses :

- purification sur grande colonne de gel d'alumine (six labos), gel de silice (18 labos) ou gel de silice traité au nitrate d'argent (cinq labos), ou sur petite colonne de silice (neuf labos) ou de Florisil (un labo);

- analyse par CPG-FID, CPG-spectrométrie de masse (SM) (trois labos) ou couplage HPLC-CPG (deux labos) ;

- calibration interne (32 labos) avec un alcane (C10, C13, C14, C15, C16, C17, C18, C20, C40, C44), du squalane, du dichlorobenzène ou du naphtalène ;

- calibration externe (14 labos) avec une huile minérale de référence (Merck ref. 1.07160, Dr Ehrenstorfer ref. 03009010, NMI ref. RIVM-Nmi-001, Fluka ref. 69246).

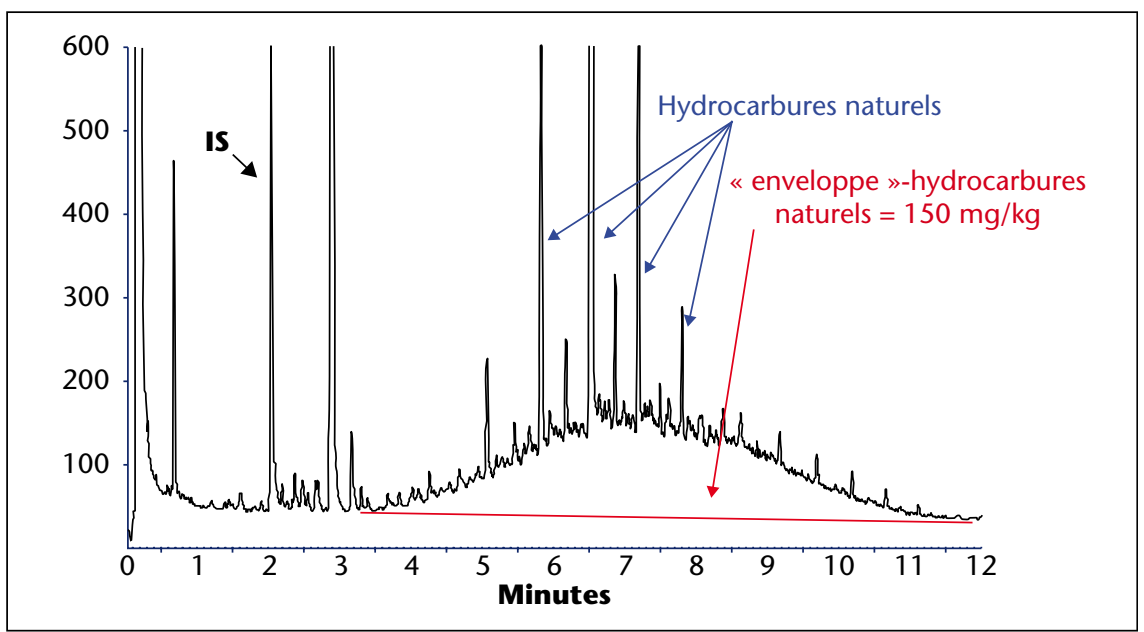

Figure 1. Chromatogramme d'une huile de tournesol contaminée en huile minérale à hauteur d'environ $150 \mathrm{mg} / \mathrm{kg}$.
Le bilan de cet essai est relativement satisfaisant : - sur 55 participants, $85 \%$ ont des résultats qui satisfont les critères de performance retenus (z-score compris entre -2 et +2 ) pour I'huile brute de tournesol contaminée en huile minérale ;

- sur 54 participants, $78 \%$ ont des résultats qui satisfont les critères de performance retenus pour I'huile raffinée de tournesol contaminée en huile minérale ;

- sur 54 participants, $83 \%$ ont des résultats qui satisfont les critères de performance retenus pour l'huile de tournesol complémentée avec I'huile minérale de référence.

Les points critiques de cette détermination concernent la calibration de l'instrument, l'intégration de l'enveloppe chromatographique due à la présence d'huile minérale et le calcul des résultats (soustraction des hydrocarbures naturels).

Au cours de l'année 2009, trois méthodes ont été proposées à I'ISO en vue de la normalisation d'une méthode sur la détermination des huiles minérales dans les huiles végétales (tableau 1). Il $s^{\prime}$ agit :

- d'une méthode française et d'une méthode espagnole couvrant toutes les deux les hydrocarbures de longueur de chaîne comprise entre C20 et C50 ;

- d'une méthode néerlandaise couvrant le domaine C10-C56.

II a été décidé au niveau de I'ISO d'explorer ces méthodes et d'essayer de les combiner dans un même texte.

\section{Phtalates}

\section{Contexte}

Le contact entre les aliments et les emballages plastiques peut être à l'origine de transferts réciproques. Les phtalates et les adipates sont utilisés comme " plastifiants " dans la production de PVC pour rendre les plastiques plus souples et plus flexibles. Le di-(2-éthylhexyl)-phtalate (DEHP) est le plus communément utilisé. Le PVC entre dans la fabrication d'objets usuels les plus divers: jouets, emballages alimentaires. Les phtalates sont également utilisés comme additifs dans l'industrie cosmétique (vernis à ongles, parfums, shampoing, laques), les produits pharmaceutiques, les encres d'impression, les enduits d'étanchéité ou les adhésifs.

Des articles parus dans la presse allemande témoignent de la présence de DEHP, de di-isodécyl-phthalate (DIDP) et de butylbenzyl-phthalate (BBP) dans certaines huiles d'olive vierges, avec des teneurs pouvant dépasser quelques dizaines de milligrammes par kilogramme. D'autres huiles vierges sont également concernées, comme les huiles de noix. Dans ces cas de figure, il pourrait s'agir 
Tableau 1. Méthodes proposées à la normalisation pour la détermination de la teneur en huiles minérales dans une huile végétale.

\begin{tabular}{|llll|}
\hline Méthode & Proposition des Pays-Bas & Proposition de l'Espagne & Proposition de la France \\
\hline Prise d'essai & $1 \mathrm{~g}$ & $1 \mathrm{~g}$ & $2 \mathrm{~g}$ \\
Adsorbant & Oxyde aluminium & Gel de silice traité au nitrate d'argent & Cel de silice \\
Étalon interne & Solution de $\mathrm{C} 10$ & solution de $\mathrm{C} 20$ & Solution de $\mathrm{C} 18$ \\
Solvant pour la purification & $\mathrm{n}$-pentane $(50 \mathrm{~mL})$ & $\mathrm{n}$-hexane $(55 \mathrm{~mL})$ & $\mathrm{n}$-hexane $(150 \mathrm{~mL})$ \\
CPG-FID & $\mathrm{DB} 5(30 \mathrm{~m}-0,25 \mathrm{~mm}-0,25 \mu \mathrm{m})$ & $\mathrm{DB} 5(15 \mathrm{~m}-0,32 \mathrm{~mm}-0,1 \mu \mathrm{m})$ & $\mathrm{DB} 5 \mathrm{HT}(15 \mathrm{~m}-0,25 \mathrm{~mm}-0,1 \mu \mathrm{m})$ \\
Programme thermique & $55^{\circ} \mathrm{C}(2 \mathrm{~min}) \rightarrow 330^{\circ} \mathrm{C}(10 \mathrm{~min}) \mathrm{à} 10^{\circ} \mathrm{C} / \mathrm{min}$ & $50^{\circ} \mathrm{C}(1 \mathrm{~min}) \rightarrow 350^{\circ} \mathrm{C}(4 \mathrm{~min})$ à $12{ }^{\circ} \mathrm{C} / \mathrm{min}$ & $100^{\circ} \mathrm{C} \rightarrow 370^{\circ} \mathrm{C}(2 \mathrm{~min})$ à $20^{\circ} \mathrm{C} / \mathrm{min}$ \\
\hline
\end{tabular}

de migration des phtalates des emballages plastiques ayant contenu les fruits avant pressage ou des différents matériels (bandes de convoyage, tuyaux plastiques, etc.) utilisés lors du process.

L'ITERG pour cette raison est en cours de validation d'un protocole d'analyse de ces composés pour en surveiller la teneur dans des huiles alimentaires.

Aucune spécification réglementaire européenne ne fixe de teneurs limites dans les huiles alimentaires et les corps gras. Toutefois, I'annexe III de la directive 2007/19/CE précise que l'usage des phtalates comme plastifiants est réservé à des matériaux en contact avec des aliments non gras, ce qui revient à dire qu'ils ne peuvent être employés dans la fabrication de matériaux destinés au contact d'aliments gras.

\section{Méthodes d'analyse}

II n'existe pas encore de méthode à l'étude au niveau de la normalisation sur ce sujet, cependant l'analyse des phtalates dans les huiles alimentaires a fait l'objet de publications (Di Bella et al., 2004; Dugo et al., 2004 ; Mariani et al., 2006 ; Fusari, 2009, Cavaliere et al., 2008) et de nombreuses équipes travaillent actuellement sur cette thématique, dont notamment I'ITERG.

La méthode développée par I'ITERG repose sur une microextraction sur phase solide (SPME) couplée à une quantification par CPG couplée à la spectrométrie de masse (CPG-SM). Un échantillon de $1 \mathrm{~g}$ d'huile est chauffé à $160^{\circ} \mathrm{C}$ dans un flacon de $10 \mathrm{~mL}$ et une fibre recouverte de différents adsorbants (PDMS-CAR-DVB ; 30/50 $\mu \mathrm{m}$ d'épaisseur) est introduite dans l'espace de tête de ce flacon durant 25 minutes. Les composés adsorbés sur la fibre sont ensuite volatilisés lors de l'introduction de la fibre dans l'injecteur du CPG-SM. Cette méthode présente l'avantage de ne pas nécessiter de préparation préalable des échantillons. De plus, aucun solvant n'est utilisé pour extraire les phtalates ce qui est un point positif pour l'environnement et pour la santé des opérateurs.

Les limites de détection et de quantification des di-butyl-phtalate (DBP), benzyl-butyl-phtalate (BBP), DEHP et di-(n-octyl)-phtalate (DNOP) sont inférieures à $1 \mathrm{mg} / \mathrm{kg}$. Pour les di(isononyl)phtalate (DINP) et di-(isodécyl)-phtalate (DIDP), la mauvaise résolution chromatographique induit une limite de quantification plus élevée.

Des méthodes plus sophistiquées, basées sur l'extraction liquide-liquide, puis la purification sur adsorbant suivie de l'analyse par CLHPSM-SM, permettent de quantifier plus aisément les DINP et DIDP (Dugo et al., 2004).

\section{Esters de}

\section{3-monochloropropane-1,2-diol}

\section{Contexte}

Le 3-monochloropropane-1,2-diol, ou 3-MCPD libre, a été identifié dans les années 1980 dans des échantillons de protéines végétales ayant subi une hydrolyse acide. Sa présence a également été constatée dans divers aliments, tels que les sauces de soja, le pain, les toasts, les crackers, le malt, le fromage grillé, certains produits carnés. II semblerait que le mécanisme de formation du 3-MCPD soit favorisé par des procédés thermiques en présence de glycérol ou d'acylglycérols et d'ions chlorures.

L'activité génotoxique du 3-MCPD a été démontrée par des études in vitro. II est cancérigène chez les rats et présente une toxicité lors de la reproduction. La dose journalière admissible (DJA) a été fixée par le JECFA à $2 \mu \mathrm{g} / \mathrm{kg}$ de poids corporel et la Commission européenne limite la teneur en 3-MCPD à $0,02 \mathrm{mg} / \mathrm{kg}$ pour la sauce de soja et les protéines végétales hydrolysées (Règlement $C E, 2006)$. Une méthode de dosage dans les aliments du 3-MCPD libre fait l'objet d'une norme EN 14573 datant de 2005 (EN, 2005). Récemment, la présence de 3-MCPD sous forme de diesters de 3-MCPD (figure 2) a été signalée dans des huiles végétales raffinées (Zelinkova et al., 2006 ; Weisshaar, 2008) ainsi que dans des produits alimentaires formulés contenant des matières grasses végétales. Les professionnels de la filière sont questionnés par leurs clients quant à la maîtrise de ce contaminant potentiel et souhaitent disposer d'éléments complets et fiables sur cette question. Les premiers résultats sur la présence d'esters de 3-MCPD dans les aliments datent de 2004 (Dolezal et al., 2005 ; Svejkovska et al., 2004). Les aliments concernés sont tous issus de procédés où intervient une étape de chauffage : frites, pain grillé, crackers, café et malt torréfiés, saucisse, lait maternisé.

En 2006, l'équipe de Velizek montre que la formation d'esters de 3-MCPD dans les huiles serait une conséquence de l'étape de désodorisation et que des teneurs élevées $(2,4 \mathrm{mg} / \mathrm{kg})$ peuvent être détectées dans les huiles raffinées (Cavaliere et al., 2008).

Les travaux de Weisshaar (2008) sur plus de 400 échantillons d'huiles confirment ce résultat. Les huiles vierges ne contiennent pas d'esters de 3-MCPD $(<0,1 \mathrm{mg} / \mathrm{kg})$, alors que les huiles raffinées contiennent entre 0,2 et $21 \mathrm{mg} / \mathrm{kg}$ avec une médiane à $0,9 \mathrm{mg} / \mathrm{kg}$.

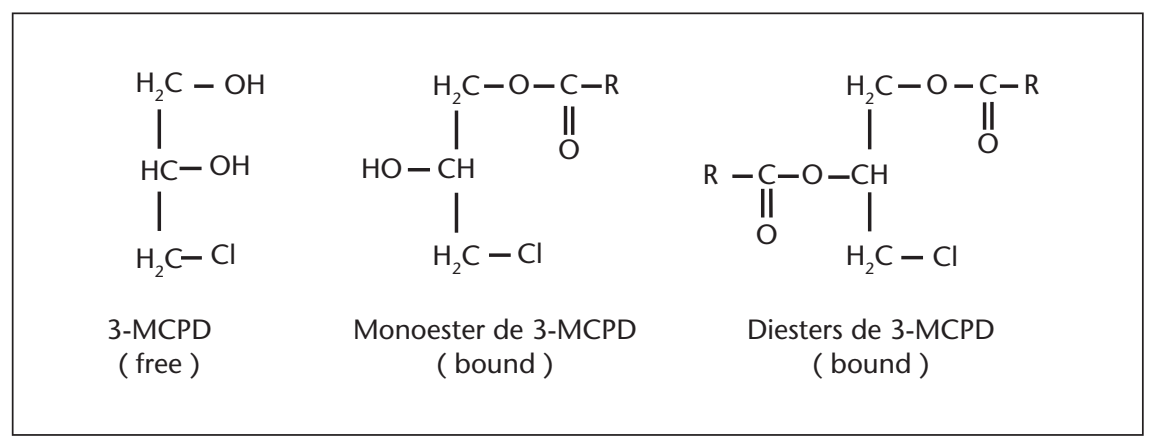

Figure 2. Structure du 3-MCPD libre et des mono- et di-esters de 3-MCPD. 
Les huiles à risque seraient plus particulièrement les huiles et les fractions de palme, les huiles hydrogénées ou interestérifiées (car double raffinage). Les margarines seraient également concernées et, en fait, tous les produits contenant de I'huile de palme. II semblerait qu'au cours d'un chauffage prolongé (11 heures) à des températures de l'ordre de $150-180^{\circ} \mathrm{C}$, la teneur en esters de 3-MCPD diminue, ce qui corrobore les résultats obtenus sur des huiles avant et après friture collectées dans des restaurants.

Cependant, tous ces résultats ont été obtenus avec une méthode qui pourrait conduire à des résultats surestimés.

En l'absence d'étude poussée sur les effets sur la santé des esters de 3-MCPD, ou sur la part de 3-MCPD pouvant être libérée par les esters au cours des processus digestifs, il est impossible de se prononcer sur le risque encouru par la présence de ces esters dans les aliments. Le 28 mars 2008, I'EFSA a rendu un avis sur la nécessité de poursuivre des études relatives à la libération des esters de 3-MCPD in vivo (EFSA, 2008b).

En février 2009, à l'initiative de I'International Life Sciences Institute (ILSI), de la Commission européenne et de l'EFSA, un workshop intitulé $3 M C P D$ esters in food products a été organisé à Bruxelles. II regroupait les principaux experts industriels et institutionnels européens (chimistes analystes, toxicologues, technologues, nutritionnistes, etc.). Cet atelier avait pour objectif de faire l'état de l'art sur ce contaminant, de rappeler les positions institutionnelles et d'identifier les domaines d'investigation; son compte rendu est disponible sur Internet (International Life Science Institute, 2009).

D'un point de vue général, il est ressorti de ce workshop la nécessité de poursuivre les travaux sur les esters de 3-MCPD (et les glycidol-esters), sujet qui doit être considéré comme un problème potentiel de santé publique. Plus spécifiquement, les sujets suivants sont considérés comme prioritaires :

- disposer de méthodes analytiques validées pour la quantification individuelle de chaque famille : 3-MCPD libre, monoesters, diesters, chlorés en position 2 ou 3 et les esters de glycidol, dans les huiles et aliments gras ;

- disposer d'une méthode de dosage des esters de 3-MCPD dans les fluides biologiques et notamment le plasma (méthode indispensable à l'évaluation de la biodisponibilité de ces contaminants);

- comprendre les mécanismes de formation des esters de 3-MCPD et des esters de glycidol (si mécanismes indépendants) au cours du process (trituration, raffinage, hydrogénation, etc.) afin d'envisager des stratégies de réduction de ces composés ;
- compléter les bases de données des teneurs en 3-MCPD et en diesters de 3-MCPD dans les aliments ;

- évaluer et comparer la toxicité in vivo chez l'animal de molécules de synthèses pures: 3-MCPD libre, diesters de 3-MCPD et monoesters de 3-MCPD (sn1 et sn2) ;

- déterminer l'efficacité des mécanismes physiologiques de digestion-absorption des différents esters de 3-MCPD afin de comprendre leur toxicité éventuelle dans l'organisme.

\section{Méthodes d'analyse}

À ce jour, seule I'Allemagne, via la DGF, son institut de normalisation pour l'analyse des huiles, corps gras et lipides, a validé une méthode d'analyse du 3-MCPD dans les huiles végétales et les matières grasses extraites des produits transformés (DGF, 2008). Elle repose sur un clivage des esters de glycérol en présence de méthoxyde de sodium puis une dérivatisation du 3-MCPD libre, endogène et libéré à partir des esters, par l'acide phénylboronique. Les dérivés obtenus sont analysés par CPG-SM ou CPG-SM-SM. La quantification est réalisée par l'ajout d'un étalon interne, le 3-MCPD deutéré, et la réalisation d'une courbe de calibration. La teneur en esters de 3-MCPD est alors exprimée en équivalent 3-MCPD libéré.

Bien que publiée, cette méthode fait l'objet de nombreuses critiques de la part de la communauté scientifique. Le principal reproche est l'utilisation d'une solution très concentrée en chlorure de sodium $(20 \mathrm{~g} / \mathrm{L})$ à la fin de la première étape. II en résulte une forte concentration en ions chlorures qui seraient susceptibles de générer du 3-MCPD en réagissant avec le glycidol (figure 3) présent dans les huiles, lors de la dérivatisation à l'acide phénylboronique. En effet, la dérivatisation à l'acide phénylboronique est réalisée à $80^{\circ} \mathrm{C}$. Cette réaction au cours de la préparation de l'échantillon conduirait ainsi à des valeurs surestimées de 3-MCPD.

Cette méthode indirecte présente d'autres inconvénients; notamment, la répartition entre monoesters et diesters n'est pas mesu- rable, ce qui limite l'étude des réactions pouvant avoir lieu au cours des différentes étapes du raffinage.

Très peu de publications évoquent la possibilité de doser les esters de 3-MCPD sous leur forme native (Seefelder et al., 2008 ; Collison, 2010). Elles nécessitent, soit de fractionner l'échantillon sur une ou plusieurs cartouches d'adsorbant en vue de s'affranchir des triglycérides, soit d'utiliser un outil d'analyse sophistiqué de type couplage HPLC-ESI-TOF-MS permettant une analyse directe de l'huile.

Cependant, aucune donnée de validation interlaboratoire de ces méthodes n'est fournie à ce jour. L'une des principales limites au développement d'une méthode directe est l'absence des étalons de référence et de quantification (deutérés si possible).

\section{Conclusion}

Le contrôle des huiles végétales au niveau industriel montre que leur contamination est très faible et qu'elles respectent les limites fixées par la réglementation européenne. Cependant, l'utilisation d'outils analytiques de plus en plus puissants peut conduire à la détection dans les aliments de nouvelles molécules pouvant présenter un risque pour la santé. Ce fut le cas de l'acrylamide, puis récemment des esters de 3-MCPD et des esters de glycidol. Afin de mener des études toxicologiques nécessaires pour fixer des limites réglementaires, le développement de méthodes de dosage justes, robustes et validées est essentiel. Cet objectif est une des missions prioritaires de l'ITERG.

\section{RÉFÉRENCES}

EFSA. Avis du groupe scientifique sur les contaminants de la chaîne alimentaire (2008a), « Les hydrocarbures aromatiques polycycliques dans I'alimentation ». The EFSA J 2008: 724. http://www.efsa.europa.eu/fr/ scdocs/doc/s724fr.pdf.

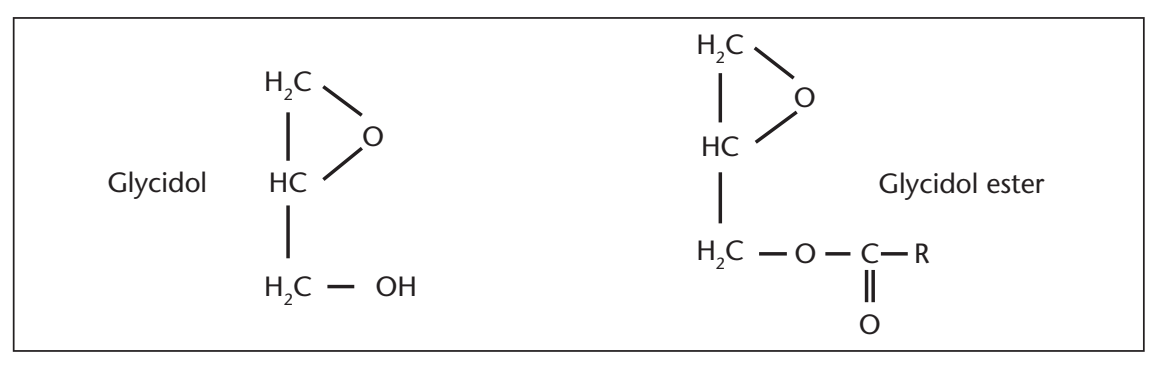

Figure 3. Structure du glycidol libre et des mono-esters de glycidol. 
Cavaliere B, Macchione B, Sindona G, Tagarelli A. Tandem mass spectrometry in food safety assessment: the determination of phthalates in olive oil. J Chromatogr A 2008 ; 1205 : 137-43.

Collison MW. Direct determination of MCPD esters and glycidyl esters by LCMS, OVID Meeting, 25 January 2010, Berlin, 2010.

DGF Standard Method C-III 17b. Determination of ester-bound 3-chloropropane-1,2-diol (3-MCPD esters) and 3-MCPD forming substances in fats and oils by means of GC-MS, 2008.

Dolezal M, Chaloupska M, Divinova V, Svejkovska B, Velisek J. Occurrence of 3-chloropropane-1,2-diol and its esters in coffee. Eur Food Res and Technol $2005 ; 221: 221-5$.

Di Bella G, Condoleo C, Bruzzese A, Salvo F, Cappello A, Dugo G. Residui di plastificanti in oli vegetali-Nota I. La Rivista Italiana delle Sostanze Grasse 2004 ; 81 : 207-10.

Directive CE 2004/73/CE de la commission du 29 avril 2004 portant sur la $29^{\mathrm{e}}$ adaptation au progrès/technique de la directive 67/548/CEE du Conseil concernant le rapprochement des dispositions législatives, réglementaires et administratives relatives à la classification, l'emballage et l'étiquetage des substances dangereuses.

Dugo G, Di Bella G, Saitta M, Conoleo C, Lo Turco V. Residui di plastificanti in oli vegetali-Nota II. La Rivista Italiana delle Sostanze Grasse 2004 ; 81 : 273-6.

EFSA. Statement of the scientific panel on contaminants in the food chain (CONTAM) on a request from the European Commission related to 3-MCPD esters, Question N ${ }^{\circ}$ EFSA-Q-2008-258, 2008b. http://www. efsa.europa.eu/fr/scdocs/doc/1048.pdf.

EN 14573. Produits alimentaires-dosage du 3-monochloropropane-1,2-diol par CG/SM, 2005.

European Commission. Opinion of the Scientific Committee on Food on the risks to human health of polycyclic aromatic hydrocarbons. Health and Consumer Protection Directorate General, 4 December 2002. http:// europa.eu.int/comm/food/fs/sc/scf/index_en.htlm.

Fiume F, Ferrieri F, Froio G, Spinelli S, Lattarulo O, Fanuzzi G. Determinazione di idrocarburi policiclici aromatici in oli alimentari. La Rivista Italiana delle Sostanze Grasse 2002 ; 79 : 151-5.

Fusari P, Rovellini P. Liquid chromatography-lon Trap-ESI-mass spectrome-try in food safety assessment: phthalates in vegetable oils. La Rivista Italiana delle Sostanze Grasse 2009 ; 86 : 25-30.
International Life Science Institute. Summary Report of a Workshop held in February 2009 in Brussels. Belgium : ILSI Europe Report Series, 2009. http://www.ilsi.org/ Europe/Publications/Final\%20version\%203\% 20MCPD\%20esters.pdf.

ISO 15302. Corps gras d'origines animale et végétale. Détermination de la teneur en benzo[a] pyrène. Méthode par chromatographie en phase liquide à haute performance à polarité de phase inversée, 2007.

ISO 15753. Corps gras d'origines animale et végétale. Détermination des hydrocarbures aromatiques polycycliques, 2006.

ISO 22959. Corps gras d'origines animale et végétale. Détermination de la teneur en hydrocarbures aromatiques polycycliques par chromatographie de complexe donneur-accepteur et CLHP avec détection par fluorescence, 2009.

Karasek L Wenzl T, Ulberth F. Proficiency test on the determination of mineral oil in sunflower oil. Final report European Commission, Joint Research Centre, Institute for Reference Materials and Measurements, EUR 23811EN-2009, 2009.

Règlement CE $n^{\circ} 1881 / 2006$ de la commission du 19 décembre 2006 portant fixation de teneurs maximales pour certains contaminants dans les denrées alimentaires. http://eur-lex.europa.eu/LexUriServ/LexUriServ.do?uri=O]:L:2006:364:0005:0024: FR:PDF.

Mariani C, Venturini S, Grob K. Sulla presenza di ftalati negli oli vegetali. La Rivista Italiana delle Sostanze Grasse 2006 ; 83 : 251-6.

McGill AS, Moffat CF, Mackie PR, Cruickshank P. The composition and concentration of $n$-alkanes in retail samples of edible oils. / Sci Food Agric 1993; 61 : 357-62.

Moreda W, Rodriguez-Acuna R, Perez-Camino MC, Cert A. Determination of heavy polycyclic aromatic hydrocarbons in refined olive pomace and othervegetable oils. / Sci Food Agric 2004 ; 84 : 1759-64.

Moret S, Conte LS. A rapid method for polycyclic aromatic hydrocarbons determination in vegetable oils. / Separation Sci $2002 ; 25$ : 96-100.

Moret S, Populin T, Conte LS, Grob K, Neukom HP. Occurrence of C16-C45 mineral paraffins in olives and olive oils. Food Addit Contam 2003; 20: 417-26.
Neukom HP, Grob K, Biedermann M, Noti A. Food contamination by $\mathrm{C} 20-\mathrm{C} 50$ mineral paraffins from the atmosphere. Atmos Environ 2002 ; 36 : 4839-47.

Règlement $C E \mathrm{n}^{\circ}$ 1151/2009 de la commission du 27 novembre 2009 soumettant l'importation d'huile de tournesol originaire ou en provenance $d^{\prime} U k$ raine à des conditions particulières, en raison d'un risque de contamination par des huiles minérales, et abrogeant la décision 2008/433/CE.

Reich AG, Waylett DK, Van Der Reit E, et al. Intake of naturally occurring alkanes. Prepared for the American Petroleum Institute by TAS-ENVIRON, 1997. http://www.inchem.org/documents/jecfa/jecmono/ v50je04.htm.

Directorate-General Health and Consumer Protection (DG SANCO). Reports on tasks for scientific cooperation, "Collection of occurrence data on polycyclic aromatic hydrocarbons in food", EU Scoop Task (2004). http://ec.europa.eu/food/food/chemicalsafety/contaminants/scoop_3-2-12_final_report_pah_en.pdf

Seefelder W, Varga N, Studer A, Williamson G, Scanlan FP, Stadler RH. Esters of 3-chloro1,2-propanediol (3-MCPD) in vegetable oils: significance in the formation of 3-MCPD. Food Addit Contam Part A $2008 ; 25$ : 391-400.

Svejkovska B, Novotny O, Divinova V, Reblova Z, Dolezal $M$, Velisek J. Esters of 3-chloropropane1,2-diol in foodstuffs. Czech J Food Sci 2004; 22 : 190-6.

Tan YA, Kuntom A. Gas chromatographic determination of hydrocarbons in crude palm kernel oil. / $A O A C$ Int $1993 ; 76: 371-6$.

Van Stijn F, Kerkhoff MAT, Vandeginste BGM. Determination of polycyclic aromatic hydrocarbons in edible oils and fats by on-line donor-acceptor complex chromatography and high-performance liquid chromatography with fluorescence detection. / Chromatogr A $1996 ; 750: 263-73$.

Wagner C, Neukom HP, Grob K, Moret S, Populin T, Conte LS. Mineral paraffins in vegetable oils and refinery by-products for animal feeds. Mitt Lebensmittelunters Hyg $2001 ; 92: 499-514$.

Weisshaar R. Determination of total 3-chloropropane-1,2-diol (3-MCPD) in edible oils by cleavage of MCPD esters with sodium methoxide. Eur / Lipid SCi Technol 2008 ; 110 : 183-6.

Zelinkova Z, Svejkovska B, Velisek J, Dolezal M. Fatty acid esters of 3-chloropropane-1,2-diol in edible oils. Food Addit Contam Part A 2006 ; 23 : 1290-8. 